\title{
Implement User-Centered Design in Internationally Distributed Design Teams
}

\author{
Limin Fu and Stephanie Chan \\ Alibaba.com Inc., 3945 Freedom Circle Suite 600, Santa Clara, California, USA \\ \{paulfu, stephaniechan\}@alibaba-inc.com
}

\begin{abstract}
With globalization, international business expansion and acquisition, business process outsourcing, distributed design teams become a common practice in many companies and organizations. Many challenges exist for distributed teams. Research conducted in the past looked into communication, project management, and organizational and people management to overcome those challenges. This paper provides a case study, which not only leveraged the findings provided in the previous research, but also looked into the advantages and disadvantages within each user-centered design role in distributed locations. The organizational, project, and role arrangement in distributed teams were discussed with emphasis on how to implement user-centered design in internationally distributed design teams.
\end{abstract}

Keywords: Distributed Team, Design Management, User-centered Design.

\section{Introduction}

Distributed design teams become a common practice in many companies and organizations with globalization. Companies and organizations add internationally distributed teams through international business expansion, acquisition, and outsourcing. Technology advances further reduced the communication cost and enabled such business arrangement.

\section{$2 \quad$ Literature Review}

\subsection{Advantages of Distributed Teams}

Benefits of internationally distributed teams are apparent. In many cases, distributed teams are not only to reduce time-to-market and development cost [7] but can also promote diverse knowledge, local expertise, and discovery of innovative solutions from the diversity in the different cultures, background and language [1, 13, 3, 7]. Members at different sites are likely to use different knowledge sources and networks to search for solutions [3]. This arrangement will allow for individuals to work with different people through various projects, create opportunities in knowledge sharing and "cross-pollination of ideas among different groups" [13]. The internationally 
distributed teams bring opportunities and competitive advantage to companies and organizations.

Challenges in Distributed Design Team. While diversity can bring new perspectives, it can also cause conflicts in the differences in work ethics, cultural perspectives and use of processes and tools [7]. Thus, distributed teams are not without its challenges - challenges in diversity, distance, technology, and communication can threaten to fragment the team [3].

Communication Challenges. For distributed teams, communication is a key factor but the ability to achieve effective design communication is immensely difficult often due to the restraints in collaborative tools. Distributed teams face intensified mental workload, physical demand, and frustration as correspondence becomes restrained from face-to-face to video to audio [6]. Collaborative tools often make communication become less natural and fluid as well as cause disturbances in the creative and social processes for the design team [1]. These disturbances include the lost in body language, gestures, gazes, and eye contact [1]. As Bergstrom and Torlind concluded from their comparison between a co-located and distributed design session, "if the communication flow is to be more natural, such as in the co-located setting, it could also give greater support to embodied representations and potentially support the flow of a bodystorm" [1]. Another unfortunate lost is the informal side conversations or water cooler discussions, which can be crucial in building on the ideas of others [1] and the development in mutual understanding of projects at a faster pace $[1,14,13]$. Such restraints cause group interaction to decrease as it becomes less likely to discuss issues and alternatives as they arise and will often wait until they face a critical issue before they interact [6].

Time Zone Challenges. Depends on the location of the distributed team, the time zone challenge is different. In some cases, teams are located in a place with large time zone differences, for example, U.S. and China. While there are many tools available for brainstorming and facilitating design sessions, it is not a sustainable model to have design teams frequently collaborate in the office over video conference with 16 hours of time difference.

Outsourcing Model Challenges. In most cases, outsourcing within the organization would not work in the long term. In such cases, teams are located in different economics zones where one location is much more expensive than the other. It may seem like a good idea to leverage an outsourcing model by focusing the more expensive team on more highly creative and value-adding activities, and have the less expensive team work on routine and less value-adding services, such as picture cropping and banner creation. But in the long run, there is a big challenge to motivate the less expensive team.

Cultural Differences. Team members may come from different cultures and countries. As Hofstede [8] identified, cultures could be different in several dimensions. Value, way of communication, inspiration, and team dynamics are different from one culture group to the other. Hall [5] looked at the culture differences from communication 
context point of view. For example, people with high context culture are more relational, collectivist, and indirect with emphasis on interpersonal relationships. Working with high context culture, developing trust is an important first step as the preference is in group harmony and consensus to individual achievement. People in high context cultures are less governed by reason than by intuition or ings. Words are not so important as context, which might include the speaker's tone of voice, facial expression, and gestures. In some cases, even the person's family history and status is an important context to the communication. The information flows efficiently among the group members, where discussion and decisions happen before meetings. Meeting is considered official ceremony rather than a formal decision making process. Communication between high context and low context culture might be problematic [2]. When people with low context culture provide too much information to people with high context culture, people with high context culture might feel impatient. When people with high context culture do not provide enough information to people with low context culture, people with low context culture might feel puzzled. The challenge of the communication is to find the right amount of information based on the context.

Lack of Influence. Distance in physicality for distributed teams can often cause uneven distribution of critical task-related information [3, 7], exclusion from important team decisions, and reduction in contribution opportunities causing feelings of isolation, disidentification, and uncertainty in their value within the team [3]. Separate locations, especially from different time zones, have distributed teams further strained from the inability to do on the spot verification, lack of visible progress, lack of trust from miscommunication, and failure to maintain mutual knowledge through differences in speed to access information and interpreting the meaning of silence [7].

Ways to Overcome Challenges in Distributed Design Team. Several articles looked into ways to overcome the challenges for distributed design teams. Those include communication tools, project management, leadership and people management.

Design Artifacts and Communication Tools. As companies venture into distributed teams, an important factor in its success is the use of materiality of artifacts - tools to support and/or to represent work [15]. There are two types, procedural artifacts (office memos, letters, Gantt charts, etc.) and design artifacts (sketches, models, prototypes, etc.) [1]. As Vyas, Heylen, Nijhot, and van der Veer found through their ethnographic fieldwork of design teams, the "material signals - signals in which people communicate through material artifacts, locations and their embodied actions" - are an integral part in helping different co-workers make sense of each other's collaborative activities [15]. The need for collaboration and communication tools such as project discussion forums, project file depository, real-time data exchange (file sharing and collaboration), instant messaging augmented with voice and email [14], video [6], and shared surfaces to build on ideas of others [1] are also key factors in achieving success in virtual teams. Because of geographical distribution, face-to-face time occurs only rarely. This, according to research, results in weaker social links between team-mates and leads the team to be more task-focused than socially focused. [14] If 
face-to-face meetings are feasible, meetings should be held as much as possible at the beginning of the team formation in order to bring team-mates closer and form interpersonal bonds. These meetings should focus more on relationship building than on actual business. However, with socializing, different cultural preferences have to be remembered. If face-to-face meetings are not possible or feasible to the desired extent, other approaches can be applied. Social-bonding can be done partially via electronic communication tools. Jarvenpaa's and Leidner's [10] study found that if teams communicate more socially they achieve higher trust and better social and emotional relationships. Leaders can help foster relationship building and general team building in many ways, e.g. by providing continuous feedback, listening to team members' opinions and suggestions, clearly stating the team member roles and having consistency in their leadership style. With technology and network advance, many collaboration tools are available, for example, cloud-based collaboration services, like Basecamp, Dropbox, Google Drive, Amazon Cloud Drive, AliCloud, etc. Widely available high-speed broadband at homes and offices also made the international video and audio communication a commodity through tools such as Skype, Yahoo Messenger, Alibaba Trade Manager, and WeChat, etc.

Project Management. Project management in distributed teams is most influential during the planning and development phases. Team leaders must plan regular interim deliverables to keep the global teams on track with the overall schedule [7] as well as organize and encourage regular meetings to promote the free exchange of ideas [6]. As further noted by the studies of distributed collaborative design teams conducted by Hammond, Harvey, Koubek, Compton, and Darisipudi, "the use of a facilitator or trained leader is needed to ensure participation by all members to promote effective communication and free exchange of ideas" [6]. Some type of formal discussion techniques or training must be utilized to encourage participants to interact frequently and freely explore issues and alternatives for consideration [6]. These discussions will need to be followed by a well-defined and documented process, clearly communicated and understood by the team, to act as a "shared depository of project wisdom" in order to allow resurfaced issues to be resolved quickly without reworking them [7].

Leadership and People Management. Despite these challenges, there are solutions to dissolve them through team leadership and management. Wilczynski and Jennings emphasized that "the success of virtual teams is heavily dependent on the preparation of the project leaders" [14]. Web collaboration tools are just half of the set of tool, it must be augmented by effective leadership in guiding the entire design process and close management of the design infrastructure. Teams must be trained and well organized where individual activities are clearly articulated and all are aware of the assigned responsibilities and deliverables [14]. As further noted by a series of structured interviews conducted by Hashia, Whelan, and Shewaramani, it is crucial to pay attention to project management, training, and people management in distributed teams in order to combat challenges in communication, cultural differences, and sense of being a single team [7]. People management in the form of relationship-based leadership is the most vital aspect in the success of distributed teams. As the empirical study conducted by Gajendran and Joshi reveals, high quality Leader-Member Exchange 
(LMX) in combination with frequent leader-member communication in distributed teams helps enable member influence on team decisions and foster critical team-level outcomes such as innovation [3]. LMX is a relationship-based leadership approach characterized by leaders treating followers as unique individuals developing ongoing dyadic relationships that are sustained through exchanges of materials and socioemotional resources [3]. With LMX amplified by frequent team leader communication, it creates opportunities to contribute ideas and strengthen knowledge through feedback from leaders. This positive and supportive communication atmosphere can help create an environment to build upon ideas similar to the benefits of side conversations that were being lost in distributed teams. By working and reasoning on a personal level, this allows people to interact more naturally [7] and also act as a supplement to the feelings of isolation by creating a sense of proximity, value and belonging within the team as they gain a voice in team decisions. For this to be a success, team leaders must create opportunities for all team members to be involved in team decisions [3] and must promote a sense of equality where their input is equal to everyone else's [7]. "Us versus them" mentality must be discouraged by treating both locations as a single team with single focus but separate deliverables while celebrating success jointly. To provide this sense of belonging as well as build trust and personal relationships, team leaders must create opportunities for face-to-face time by organizing visitations and exchanging of people to different locations of the distributed teams [7]. These remote team visitations accompanied by classes and seminars on culture awareness and differences among work ethics, honesty, social issues, etc. can accelerate the sense of understanding and trust [7]. Pemberton-Billing's, Cooper's, Wootton's, and North's empirical studies of two co-located and distributed design projects further emphasize the importance of face-to-face contact at certain points within the life cycle of design projects - some being a central component in the formation and maintenance of distributed community of practices [13].

\subsection{User-Centered Design}

The concept of user-centered design was widely utilized in the design field. It was included in ISO standards [9] as part of the product development process. The ISO standard outlines 6 key user-centered design principles: (i) The design is based upon an explicit understanding of users, tasks and environments; (ii) Users are involved throughout design and development; (iii) The design is driven and refined by usercentered evaluation; (iv) The process is iterative; (v) The design addresses the whole user experience; (vi) The design team includes multidisciplinary skills and perspectives.

A typical user-centered design process is illustrated in Figure 1. The process typically includes a few phases, such as understand, design, prototype, research, improve, document, and develop. The design process is iterative. For example, the design could start with concept design, followed by low-fidelity prototype, concept user interviews, design improvement based on the research findings, then redesign, high-fidelity prototype, formal lab testing, etc. The final design will be documented in design specification and go through full production development. 


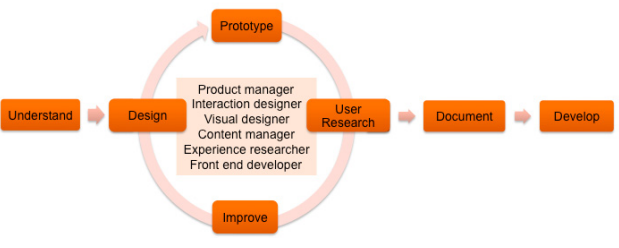

Fig. 1. A typical user-centered design process

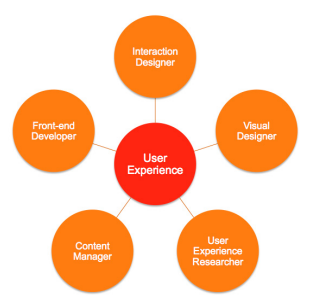

Fig. 2. Multi-disciplinary design team

The design process is a multi-disciplinary process requiring different roles to work closely. Different roles involved in this process are also illustrated in Figure 3. Product manager typically is responsible for the business side of a product, such as product planning, forecasting, and marketing in all stages of the product lifecycle. Interaction designer oversees the design process, and is responsible for understanding business requirement and user needs, design product information architecture and user interaction process. During the design process, the interaction designer, in most cases, will need to work with visual designers to come up with page details, as well as user experience researchers to understand user needs and test product usability.

Visual designer uses visual elements as the communication and expression methods. The elements include font, shape, color, graphics, and layout etc. The emotional and aesthetic aspects of the design play very important roles in the product success. User experience researcher utilizes different research methods to collect user needs, and exanimate product usability from user's point of view. While interaction and visual elements are an obvious part of design, content is also an integral part of the design process. Content manager is responsible for content strategy, communication plan, and final deliverable copies. Front-end developer is responsible for design prototype and front-end coding. Prototypes need to be developed with low cost and high speed, most of the time without backend support, in order for user experience researchers to conduct usability studies. Front-end developer is also responsible for final production of the front-end interface coding.

\section{Case Study: Implementing UCD in Distributed Teams}

With distributed teams in the U.S. and China, we faced many of the challenges illustrated in the literature review section including: communication, time zone, outsourcing model challenges, cultural differences and lack of influence. Communication tools, leadership, project and people management solutions presented in the literature review sections were helpful in combating challenges facing distributed design teams. However, in our case, with oversea distributed teams, some of the arrangements in the literature were unfit. For example, we have very advanced videoconference facilities with dual monitors and projectors, but due to the big time zone differences the usage of such office equipment is inconvenient and thus infrequent. As project collaborations increased, our teams began to adapt through trial and error and began naturally developing solutions fitting our own company culture. With that in mind, we realized 
the inefficiency and ineffectiveness in building a parallel design team in each location and forcing them to collaborate together. Instead, the advantage and disadvantage of each distributed team were identified to pinpoint how each role in different locations could contribute uniquely. Strong leadership was developed in each location to build teams with different focuses to leverage the advantage of the location - turning some of the disadvantages to unique opportunities.

Organizational Consideration. One of the natural occurrences during our collaboration process was the establishment of one-to-one discussions and dyadic relationship between leader and members within distributed teams. It was not until later that we realized that such bonding was one of the most important aspects of high quality LMX. As a result, we understood the need for leadership in globally distributed design teams to enhance team innovation and enable member influence on team decisions. As revealed by Gajendran's and Joshi's research [3], high-quality leader member exchange relationships were effective in creating member inclusion and involvement in team decisions in highly distributed settings when they were accompanied by frequent leader-member communication. We took a few steps to assess and change the organizational structure. First, we looked into the status of the team, similar to the in-group and out-group status in LMX theory. For example, the U.S. office overall was playing a consulting role, due to being separated from the main product development process and the lack of unique contribution. Geographic location and time zone difference incorporated additional hindrance to the communication.

Then, we took the effort to improve the communication, especially the dyadic communication between the leader and member. Our communication channels included video and voice conference, phone, and instant messaging tools. International travel might be costly in some cases, but in a Chinese cultural point of view, face-toface communication was a crucial and worthwhile investment in personifying the team member and building relationships. While regular group level communication was needed, frequent leader-member communication was even more important leader and member worked together to identify ways to remove these barriers. Dyadic relationship between leader and member was fostered through the effective communication. The decisional power of the leader in organizational hierarchy and the existence of transparency in the workings of organization have direct positive influence. When our leaders were moved higher in the organizational hierarchy, we had higher influence to the upper hierarchy of the organization. The team members found it satisfying and motivating. More transparency was enforced by providing detailed organizational and project information, which in turn lead to more member confidence and motivation to exceed the expectation.

Finally, we established user-centered design thinking and process within the distributed user experience team as well as the whole organization. Research showed when both leader and member have the same approach to problem solving, or cognitive similarity, high leader-member exchange level is achieved. For our design teams, user-centered design was the base guiding principle to establish some level of cognitive similarity. Presentations, seminars, and training were carried out to promote 


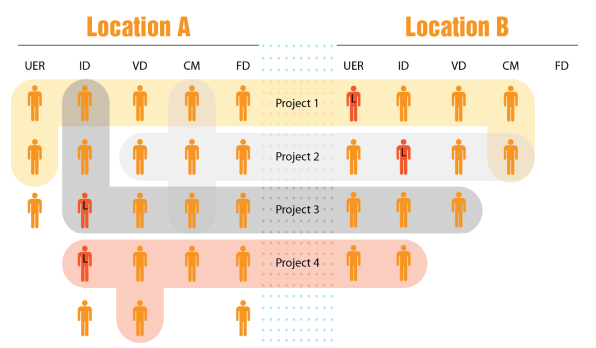

Fig. 3. Project Organization of Distributed Design Team

user-centered design within the user experience team and whole organization. The user-centered design process was integrated into the product development process: key projects would go through an iterative design, testing, and improvement cycle as the UCD process specified.

Project Organization. At project level, the distributed or virtual team concept was utilized to organize our team. Virtual team was groups of geographically, organizationally and time dispersed designers and researchers brought together by information and telecommunication technologies to accomplish one or more design and research project. For each project, a project leader was selected. Dependent on the nature of the project, people in different role can be the leader. As for a typical product design project, an interaction design lead would be more likely to work as a leader for the virtual team. The designer was responsible for carrying out the user-centered design process for the project. In the beginning of a project, face-to-face meetings were highly encouraged either through videoconferences or if feasible, international travel. The goal of those meetings were to focus more on relationship building, through bringing teammates closer together and forming interpersonal bonds, than on actual business. If face-to-face meetings were not possible or feasible to the desired extent, other approaches were applied. For example, social bonding were encouraged via electronic communication tools, such as videoconference, Skype, etc. A very important role of our project leaders was in communication and relationship building. Leaders were urged to clearly state team member roles and apply consistent leadership style as well as help foster relationship building and general team building by providing continuous feedback and listening to team members' opinions and suggestions. Culture differences had to be considered in project communication.1 For example, in high context culture, decisions were very likely to be made before a formal meeting. With this in mind, team members in low context culture would communicate with high context culture members before the meeting to influence the decision effectively.

\section{UCD Role Consideration in Distributed Teams}

Interaction and Visual Design. Two main factors were considered in deciding where to place the designers: location of project target audience and related organizational functions. Designers closest to the target user culture had the sense of context and understanding to significantly contribute in making the right design decisions. Designers from different locations were also more likely to use different source and 
network to come up with distinct and localized design ideas. The closeness to the related functions was the second factor in consideration. For example, designers located closer to the product managers could communicate more efficiently and effectively with product managers. These designers were put on the top of the consideration list for such projects. Designers were tasked with the establishment of design guidelines and appropriate design review process to ensure consistent execution across different locations. These basic design patterns were specified in a shared design pattern library to ensure all files and guidelines were easily and universally accessible. The resulting efficiency from the prevention of double or lost work allowed for more valuable time spent on studying users, understanding business goals, and project communication. Several papers mentioned about the importance of using design artifacts in distributed teams, including prototypes, demos, mockups, etc. A design review process based on those design artifacts was established to create opportunities for member contribution of ideas and knowledge sharing, creation of a positive environment to build upon ideas, and control design quality. In turn, these activities promoted the sense of member involvement and value within distributed team members.

User Experience Research. User experience research conducted internationally was a great way to leverage the distributed team. Many of the research methods, such as interviews, lab testing, and ethnographic research, etc. require face-to-face communication with users. Distributed team had easier access to local users as local offices could serve as locations to conduct such research. It was also found that researchers with similar background to the participants could identify more findings. In some cases, where participants were hard to access even with distributed offices, remote usability test tools were used. There were two types of remote testing tools: remote lab testing and quantitative automated testing. In remote lab testing, video conversation tools were used to help researchers observe and collect participants' behaviors on the screen, facial expressions, if additional cameras were available, and voice over the tool or phone. Remote quantitative testing tools allowed researchers to program test tasks with questionnaires as well as direct participants through a set of tasks using the live site, prototype, and/or mock-ups. With this tool, a large number of users could participate simultaneously from their home or office.

Content Management. With internationalization, more products needed to be supported or be marketed in more than one language. In most cases, especially with web sites, majority of interfaces were textual content making content creation an integral part in design. Similar to how the interaction and visual aspects of the interface needed design, content also needed design. Content managers were responsible for telling the story, crafting it in such a way that it resonated with the users and ideally producing an emotional response. Distributed teams closer to the target users had the advantage of understanding the context and local expertise in composing the right content or language for communication. Thus, content managers geographically and culturally closer to the target users were among the first to be considered for such projects. 
Front-End Development. Front-end development's work typically was more separated from the rest of the design team with it being more technical and instruction based. Such attributes allowed communication to be done through detailed design specification and written communication, with less of a requirement for face-to-face interactions. Placement of the team was based on the need of face-to-face interaction for more advanced and innovative projects. Location cost was another factor to consider in deciding where to place the team. It was hard to set up experiments in reality to test the effectiveness of two different team setups. We looked into other comparisons, such as attrition rate and team satisfaction, to find evidence of the effectiveness of the effort above. With the considerations and arrangement described above, the user experience team achieved higher than company average satisfaction and lower than company average attrition rate. Although it was not a direct scientific comparison, those were definitely important factors in influencing team satisfaction and attrition rates.

\section{References}

1. Bergstrom, M., Torlind, P.: Examining Creative Collaboration in Distribute and Colocated Design Teams. In: ICED 2007: 16th Int. Conf. of Engineering Design (2007)

2. Dong, J., Fu, L., Rau, P.L., Stephanidis, C., Salvendy, G.: Human-computer Interaction: User-centered Design and Evaluation, 4th edn. Tsinghua Univ. Press (2013) (in Chinese)

3. Gajendran, R., Joshi, A.: Innovation in Globally Distributed Teams: The Role of LMX, Communication Frequency, and Member Influence on Team Decisions. Journal of Applied Psychology 97(6) (November 2012)

4. Hall, E.T.: Beyond Culture. Doubleday, New York (1976)

5. Hall, E.T.: Context and meaning. In: Samovar, L.A., Porter, R.E. (eds.) Intercultural Communication: A Reader, 9th edn., pp. 34-43. Wadsworth Pub. Co., Belmont (2000)

6. Hammond, J., Harvey, C., Koubek, R., Compton, W., Darisipudi, A.: Distributed Collaborative Design Teams: Media Effects on Design Processes. International Journal of $\mathrm{Hu}-$ man-Computer Interaction 18(2) (2005)

7. Hashia, A., Whelan, J., Shewaramani, R.: An Innovative Approach to Managing Distributed Teams, Technical Report, Seattle University, Department of Computer Science \& Software Engineering, SU-CSSE-2007-2 (2007)

8. Hofstede, G., Hofstede, J.: Cultures and Organizations: Software of the Mind. McGrawHill, New York (2005)

9. Human-centred Design for Interactive System, ISO 9241-210 (2010)

10. Jarvenpaa, S., Leidner, D.: Communication and Trust in Global Virtual Teams. Journal of Computer-Mediated Communication 3(4) (1998)

11. Norman, D.: The Design of Everyday Things. Basic Books, New York (2002)

12. Norman, D.: Emotional Design: Why we love (or hate) everyday things. Basic Books, New York (2004)

13. Pemberton-Billing, J., Cooper, R., Wootton, A., North, A.: Distributed Design Teams as Communities of Practice. In: Proceedings of 5th European Academy of Design Conf. (2003)

14. Wilczynski, V., Jennings, J.: Creating Virtual Teams for Engineering Design. International Journal of Engineering Education 19(2), 316-327 (2003)

15. Vyas, D., Heylen, D., Nijholt, A., van der Veer, G.: Designing Awareness Support for Distributed Cooperative Design Teams. In: ECCE 2008 Proceedings of the 15th European Conference on Cognitive Ergonomics, vol. (6) (2008) 